\title{
The Stroboscopic Method of Speed Measurement
}

\author{
BY VAL. A. FYNN \\ Fellow, A. I. E. E. \\ Consulting Engineer, St. Louis, Mo.
}

E VERY engineer knows how difficult it is to make accurate speed measurements and how essential accuracy is in certain cases, for instance, in testing and experimental work.

In practise, speed counters and tachometers are generally used. The first register the number of revolutions made by the machine in a given time and must be employed in connection with a time measuring device, usually a stop watch. Here the sources of error are slip and the notorious inaccuracies of stop watches. The speed counter should be applied from 30 to 60 seconds, but during that time many changes can take place in the operation of the machine under test. Slip errors can be held down by the exercise of constant care or entirely eliminated by using suitable drives, but such drives cannot always conveniently be employed.

Tachometers register the rate of speed, give instantaneous records, are independent of time-measuring devices but require more or less frequent calibration and are also subject to slip errors. Tachometers of the magneto type are the most satisfactory. If regularly calibrated, used in connection with highclass voltmeters and driven by means of gears or rubber belts cooperating with sufficient $\mathrm{l}_{\mathrm{v}}^{\mathrm{r}}$ large pulleys, reasonably accurate results are secured. But it is not always feasible to drive a tachometer by means of pulleys or gears and even the best of the tachometers with perfect drives are not sufficiently accurate for all classes of work.

Tachometers of all types require an appreciable amount of power to drive them, and cannot be used for accurately measuring the speed of fractional horse power motors because they put an unknown and varying load on the motor which is a substantial percentage of the rated output of the machine and therefore materially affects the speed thereof. Speed counters are equally unsatisfactory for very small motors because they too require some power to drive them and when pressed sufficiently hard against the shaft to avoid slip, put an appreciable friction load on the motor.

One of the most difficult problems is to measure the speed of an asynchronous motor. Such measurements are primarily made with a view to ascertaining the "slip" and the difficulty arises from the fact that this slip does not amount to more than a few per cent of the synchronous speed. The speed counter is perfectly hopeless for this kind of work and the best tachometers are insufficiently accurate even when perfectly driven. In addition to this, it is seldom possible to provide any tachometer drive at all.

All these difficulties have been long understood and many attempts have been made to overcome them in a practical manner. The ideal speed measuring device must be simple enough for everyday use, must respond instantaneously to speed changes, be sufficiently accurate for all practical purposes and must not put any load on the machine under test. As far as the writer knows, stroboscopy offers the only possibility of satisfying the conditions named. Much successful work has been done along this line, for instance, by Lorenz, Benischke and particularly, Dr. Drysdale. The writer has merely followed in the footsteps of these investigators in designing and building the apparatus which, for sometime past, has been in successful use in the research and experimental department of the Wagner Electric Mfg. Co. The details of its design have been influenced by the desire to utilize as much of the apparatus already on hand as could be made use of for the purpose in view.

The outfit consists of a stroboscopic primary standard used for calibrating purposes, of a stroboscopic slipmeter for asynchronous motors, permitting of instantaneous readings, of a stroboscopic device for instantly ascertaining the speed of any kind of motor and of a number of tachometers of the magneto type. Other types of tachometers, also speed counters, are in use but are not relied upon for accurate work.

The magneto type of tachometer in conjunction with a positive drive and an accurate voltmeter, scaled to read direct in rev. per min. is used whenever possible with due regard to the limitations of this type of speed measuring device. All tachometers are frequently calibrated.

The calibrating device is based on the tuning fork. The rate of vibration of such a fork is of extraordinary accuracy, well beyond the requirements of any ordinary laboratory. According to Prof. D. C. Miller, a good tuning fork is subject to about the following variations: An increase of temperature of one deg. cent. diminishes the frequency of the fork by 0.011 per cent; an increase in the amplitude of a fork, caused to vibrate by striking it with a felt hammer, decreases the frequency by not more than 0.002 per cent for such changes of amplitude as occur in practise; an increase of the amplitude of an electromagnetically driven fork from 0.75 to 2 millimeters reduces the frequency 0.01 per cent; electromagnetic driving of a fork increases its frequency; in one case this increase was found to be 0.025 per cent.

Any periodic phenomenon, however rapid, can be seemingly arrested at any desired point by means of a stroboscope, thus if a radial line or spoke be drawn on a disk and the latter revolved at a certain speed, the line can be caused to appear stationary by observing the disk at certain intervals of time only, or illumi- 
nating it by light flashes of a certain frequency and observing same from any convenient angle. Uninterrupted observation or continuous illumination of the disk will merely show a somewhat blurred surface. Interrupted observation of the disk with the spoke can be secured by revolving another disk provided with a radial slot past a stationary shield having a corresponding slot and keeping the eye in line with the first disk and the stationary slot. The disk with the spoke will be seen every time the two slots coincide. Interrupted illumination of the disk with the spoke can be secured by substituting a source of light for the eye. The frequency of observation or illumination can be varied by varying the speed of revolutions of the moving slot or by varying the number of such slots.

If the disk with the spoke is rotated at a constant speed of, say 1000 rev. per min., the spoke will pass any fixed point 1000 times per minute and will appear stationary in space if illuminated at the rate of 1000 flashes per minute. If the flashes occur when the revolving spoke passes the 12 o'clock position, it will appear to be stationary at that point. Flashes constantly occurring one quarter of a revolution later will show the spoke at 9 or 3 o'clock, according to the direction of rotation of the disk carrying the spoke.

But other ratios of disk revolutions to number of flashes produce stroboscopic effects. As long as the disk makes one or more complete revolutions between two successive flashes, in other words, when the ratio of revolutions to flashes is $n: 1$, where $n$ is a whole number, the stationary image remains a single radial line. When the number of flashes is a multiple of the number of revolutions of the disk, the stroboscopic image is stationary, but can be a multiple of the original. Thus if a single spoke revolving at $1000 \mathrm{rev}$. per min. is viewed by the intermittent light of 3000 flashes per minute, three stationary spokes displaced by 120 degrees will be seen. While each of the three spokes is illuminated at the rate of 1000 flashes per minute, as in the case of the number of revolutions and flashes being equal, yet because the illumination produced by each flash diminishes as the number of flashes per minute increases the stroboscopic images which are a multiple of the original are less bright than those which are identical with the original.

What is true of the spoke is also true of all kinds of geometrical figures. Thus a square viewed in the light of a fixed number of flashes per minute will appear stationary and single at one set of speeds, stationary and double at another, stationary and triple at a third, and so on. A triangle, a pentagon, a hexagon, each has a set of distinctive speeds at which it appears as single, double or triple figure. Such geometrical figures are easily recognized and distinguished and since each corresponds to definite and different ratios between speed of figure and number of flashes a combination of such figures on a single disk can be made to cover a very wide range of speeds.
Since it is extremely easy to tell when a figure is stationary, stroboscopic speed measurements can be made with the same accuracy with which the tuning fork vibrates. It has been shown that this accuracy is well within $.02 \%$. But it is interesting to know the magnitude of the error which is introduced in case the reading is taken when the stroboscopic image is not stationary. If a figure-carrying disk, observed with the help of a tuning fork, departs from that speed which produces a stationary image, the latter begins to rotate, revolving in a direction opposed to that in which the disk revolves in case the speed of the latter drops, and vice versa. The more the disk "slips" the faster the figure will rotate. The number of revolutions per minute of the figure is equal to the "slip" of the disk and is a direct measure of the error introduced by taking a snap reading at a time when the stroboscopic image is moving.

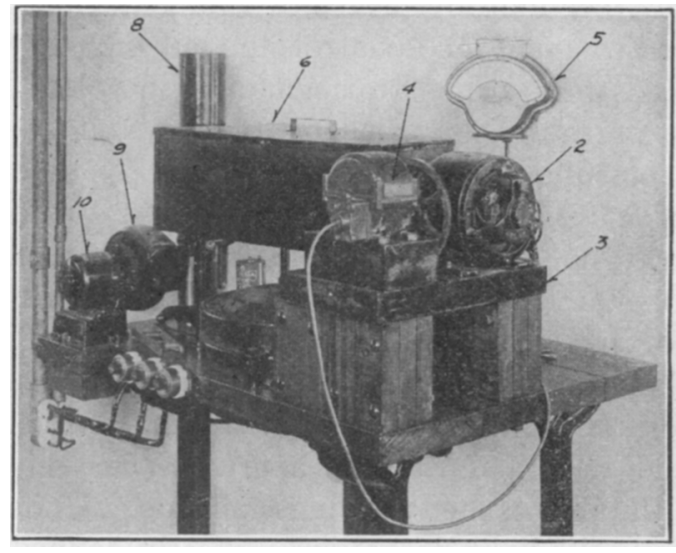

Fig. 1-Complete Calibrating Device

The complete calibrating device is shown in Fig. 1, while Figs. 2 and 3 are views of part of this apparatus. An adjustable-speed d-c. motor 2 is located on a base 3 and carries on its shaft a disk 11 upon which are displayed four concentric geometrical figures. The motors haft is geared 1 to 2 to a transverse shaft 12 which carries a pinion at one end and is cupped at the other. This shaft drives the speed measuring device which is to be calibrated. Magnetos such as 4 are driven by the pinion, tachometers with point drive are pressed against the cupped end. The voltmeter 5 in Fig. 1 is the indicator cooperating with the magneto under test. An electromagnetically driven, 50-cycle tuning fork 7 carrying a shutter near the extremity of its prongs, as well seen in Fig. 3, cooperates with a 300watt incandescent lamp located in the brass pipe 8 and so positioned as to illuminate the geometrical figures through the slits in the shutter. In order to protect the tuning fork from the heat developed by the incandescent lamp, a blower 9 driven by the motor 10 is arranged to circulate air through the pipe and a thick sheet of micanite 13 is interposed between the pipe and the fork. In order to make it possible to 
work this apparatus in the day time, a sheet iron box 6 with a removable cover and with an observation window encloses the disk carrying the geometrical figures and reaches close up to the shutter on the fork. The observation window is located at the tuning fork end. The fork is set in motion by means of the wooden pliers-like device seen in Fig. 1 just below the blower. The dry cell seen in the figures keeps the fork vibrating, the resistances shown are included in the shunt circuit of the motor and serve to adjust its speed. The finer adjustments are obtained by means of slide wire resistances mounted on the side of the box 6 not shown in Fig. 1 and are within easy reach of the operator sitting at the observation window. The various electrical circuits are controlled by means of the switches seen in Fig. 1.

The motor is a 110 -volt shunt machine of $1 / 3 \mathrm{~h} . \mathrm{p}$. at 1500 rev. per min. Its speed is varied from 100 to $4000 \mathrm{rev}$. per min. by means of voltage, armature and field circuit resistance control and no difficulty is experienced in securing stationary stroboscopic images at any of the speeds as long as the voltage of the source of supply is constant.

In order to secure clear cut images it is necessary that the disk carrying the geometrical figures run true, that the figures be concentric and that the light flashes be separated by periods of total darkness. The fork is calibrated for an amplitude of $21 / 2 \mathrm{~mm}$. and each wing of the shutter carries two slits $10 \mathrm{~mm}$. long, $0.25 \mathrm{~mm}$. wide and $7.5 \mathrm{~mm}$. apart. When the fork is at rest the slits in the two wings coincide. Under these conditions the light flashes are perfectly defined. The

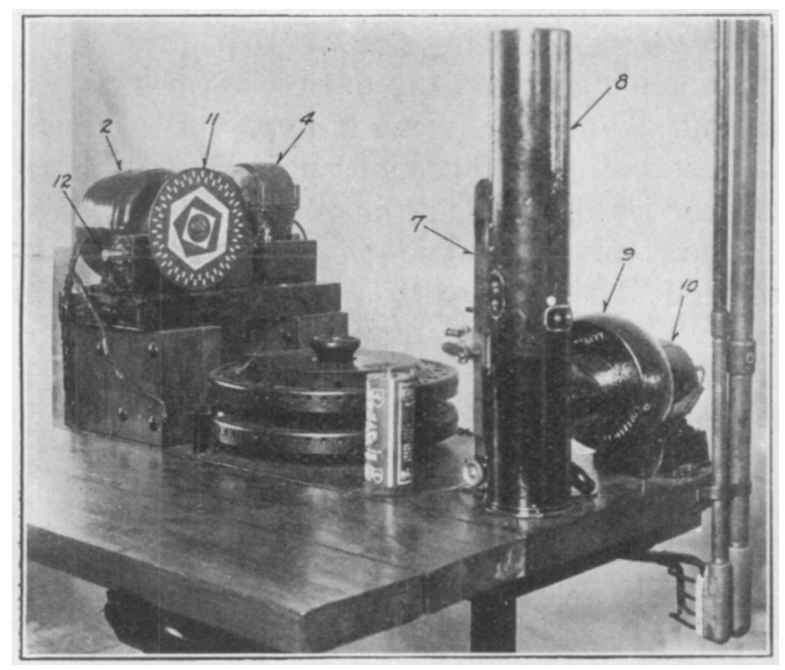

Fig. 2-Part of Calibrating Device

amplitude of the fork can be adjusted by changing the setting of the fork-operated interrupter connected in the circuit of the electromagnet which is located between the prongs of the fork. Two slits are used in order to get a more uniform illumination of the disk 11 . With a more concentrated source of light, or for viewing the disk directly through the shutter, a single and wider slot will do as well or better. One wing of the shutter can, of course, be stationary and the other attached to one prong of the fork, the latter must be calibrated at a known temperature and with the shutter in place. A 50-cycle fork produces 6000 flashes per minute.

The outer geometrical figure consists of two radially displaced rows of 30 short, equally spaced, radial lines,

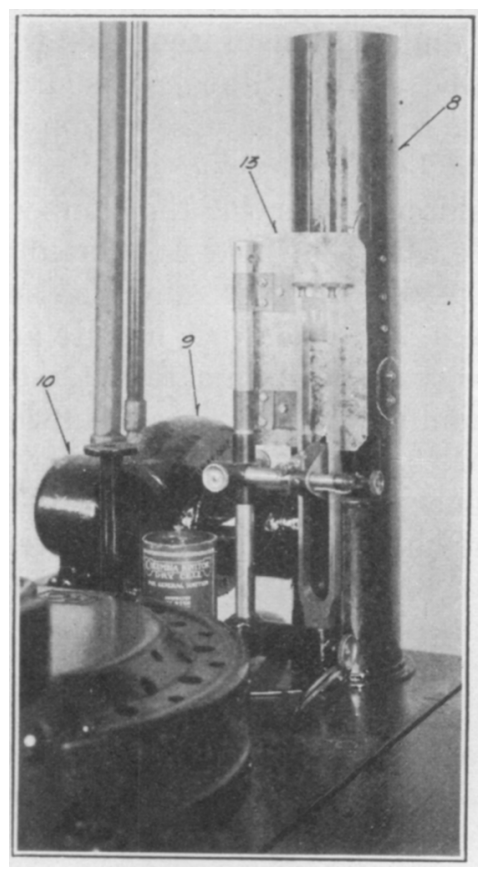

Fig. 3-Part of Calibrating Device

the 30 on one row being circumferentially displaced by six degrees from the 30 in the other row. The number of lines of each row passing a given point, each minute is thirty times the rev. per min. of the disk, or 6000 at $200 \mathrm{rev}$. per min. This figure will therefore appear stationary and single at $200 \mathrm{rev}$. per min. and all multiples thereof. At 100 rev. per min., ęach row will appear double and stationary with the result that a stroboscopic figure composed of 60 radial lines of double their true length will be seen. This will occur at 100, 300, 500 rev. per min. and so on. The sexagon will appear stationary and single at 1000 and double at 500; the pentagon stationary and single at 1200 , double at 600 , triple at 400 , and the square stationary and single at 1500 , double at 750 and triple at 500 . Single, double and triple stationary images will also be had at other speeds as shown in Table I. The speeds at which single stationary stroboscopic images appear are found by dividing the flashes per minute by the number of elements in the original figure and multiplying by any whole number as by $1,2,3,4 \ldots$. and so on. Double stationary stroboscopic figures appear at speeds found by multiplying the same quotient by any odd number of halves as by $1 / 2,3 / 2$, $5 / 2 \ldots \ldots \ldots$ and so on. Triple stationary stroboscopic figures appear at speeds found by multiplying 
said quotient by any number of thirds not divisable by three, as by $1 / 3,2 / 3,4 / 3 \ldots \ldots$ and so on.

Table I makes it plain that calibrating curves with enough points for almost any purpose can readily be obtained with the device of Fig. 1. This table is, of course, easily extended to 8000 rev. per min. which is the upper calibration limit of the apparatus. Temperature corrections should be made whenever the fork temperature during the test differs from that at which the fork was calibrated.

TABLE I

Disk speeds at which stationary stroboscopic images of various disk figures appear when viewed with a 50-cycle tuning fork. $S$ stands for single, $D$ for double and $T$ for triple image.

\begin{tabular}{|c|c|c|c|c|}
\hline \multirow[b]{2}{*}{ Disk speeds } & \multicolumn{4}{|c|}{ Disk Figures } \\
\hline & 4 point & 5 point & 6 point & 30 point \\
\hline 100 & .. & . & .. & $D$ \\
\hline 200 & .. & .. & . & $S$ \\
\hline 300 & .. & & .. & $D$ \\
\hline 400 & .. & $T$ & .. & $S$ \\
\hline 500 & $T$ & $\cdots$ & $D$ & $\dot{D}$ \\
\hline 600 & .. & $D$ & . & $S$ \\
\hline 700 & . & .. & . & $D$ \\
\hline 750 & $D$ & .. & .. & \\
\hline 800 & .. & $T$ & .. & $S$ \\
\hline 900 & & .. & & $D$ \\
\hline 1000 & $T$ & .. & $S$ & $S$ \\
\hline 1100 & .. & & .. & $D$ \\
\hline 1200 & .. & $S$ & . & $S$ \\
\hline 1300 & .. & .. & .. & $D$ \\
\hline 1400 & .. & .. & .. & $S$ \\
\hline 1500 & $S$ & .. & $D$ & $D$ \\
\hline 1600 & .. & $T$ & .. & $S$ \\
\hline 1700 & .. & .. & .. & $D$ \\
\hline 1800 & .. & $D$ & . & $S$ \\
\hline 1900 & & & & $D$ \\
\hline 2000 & $T$ & $T$ & $S$ & $S$ \\
\hline 2100 & .. & .. & .. & $D$ \\
\hline 2200 & .. & .. & .. & $S$ \\
\hline 2250 & $D$ & .. & .. & \\
\hline 2300 & .. & & .. & $\ddot{D}$ \\
\hline 2400 & .. & $S$ & .. & $S$ \\
\hline 2500 & $T$ & .. & $D$ & $D$ \\
\hline 2600 & .. & .. & .. & $S$ \\
\hline 2700 & . & & . & $D$ \\
\hline 2800 & .. & $T$ & .. & $S$ \\
\hline 2900 & .. & $\cdots$ & .. & $D$ \\
\hline 3000 & $S$ & $D$ & $s$ & $S$ \\
\hline 3100 & .. & & .. & $D$ \\
\hline 3200 & .. & $T$ & .. & $S$ \\
\hline 3300 & .. & .. & . & $D$ \\
\hline 3400 & .. & .. & . & $S$ \\
\hline 3500 & $T$ & & $D$ & $D$ \\
\hline 3600 & . & $S$ & . & $S$ \\
\hline 3700 & & .. & . & $D$ \\
\hline 3750 & $D$ & .. & . & \\
\hline 3800 & .. & . & . & $S$ \\
\hline 3900 & . & & & $D$ \\
\hline 4000 & $T$ & $T$ & $S$ & $S$ \\
\hline 4100 & .. & .. & . & $D$ \\
\hline 4200 & .. & $D$ & .. & $S$ \\
\hline 4300 & .. & & . & $D$ \\
\hline 4400 & .. & $T$ & $\ldots$ & $S$ \\
\hline 4500 & $S$ & .. & $D$ & $D$ \\
\hline
\end{tabular}

The stroboscopic slipmeter is illustrated in Figs. 4 and 5 ; it comprises a synchronous self-starting motor 15 controlled by the switch 25 and mounted on a base 14 provided with handles. This base supports a cone 16 driven by the motor through mitre gears, a stationary rod 17 and a revoluble threaded rod 20 controlled by the milled head 21 . Slidably mounted on the rod 17 is a carriage 26 which supports a disk 18 mounted on ball bearings and provided with a segmental opening, also a fixed shield 19 provided with a corresponding opening and an indicator disk 22. The disk 18 is adapted to be driven frictionally by the cone. A nut cooperating with the screw 20 engages the carriage 26 and moves it longitudinally when the screw is turned by turning the milled head 21. The travel of this nut is limited in both directions by suitably placed stops

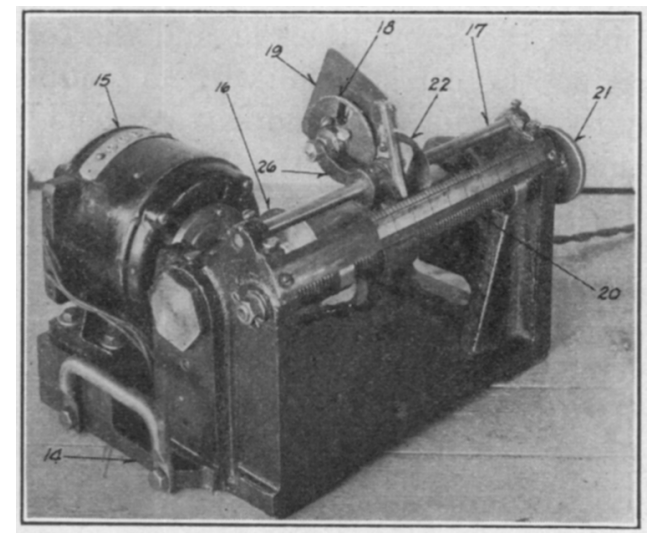

Fig. 4-View of the Stroboscopic Slipmeter

best seen in Fig. 5 which also shows the catch provided for holding the disk 18 out of contact with the cone. The position of the disk relatively to the cone is shown on the scale 24 by the indicator 22 , the zero of the scale corresponding to that disk position in which its speed equals that of the cone. This is the case when the disk contacts with a cone circumference equal to its own. The cone, disk and traversing gear are protected

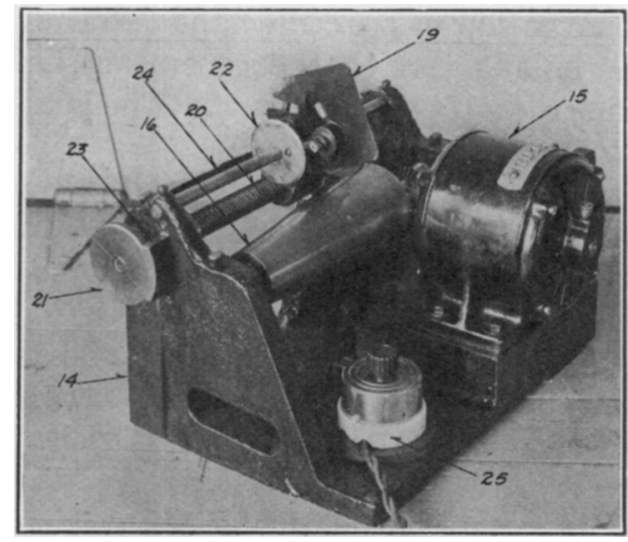

Fig. 5 -View of the Stroboscopic Slipmeter

from mechanical injury by a cover not shown in the photographs.

In using this apparatus for measuring the slip of asynchronous motors, a suitable figure is painted on a shaft end of the motor under test, the synchronous motor 15 is connected in parallel with the asynchronous machine, the disk 18 is driven by the synchronously revolving cone and the figure on the shaft end is observed through the slot in the shield 19. To measure 
a slip the milled head 21 is turned in the one or the other direction until the figure on the shaft appears stationary, when the scale is read. The scale is not only arranged to read direct in per cent slip, but the diameter of the disk 18 and the angle of the cone are so chosen that one revolution of the milled head 21 corresponds to 1 scale division or to one per cent slip. The $2 \frac{1}{4}$ in. milled head is divided into 20 parts, thus making it possible to read to $1 / 20$ th and estimate to $1 / 100$ of a per cent. As it would be practically impossible to make the screw, the nut and the fork of the nut engaging the carriage 26 without endplay, it is necessary to allow for such endplay as exists by handling the milled head 21 like the dividing head on any tool. The final position must be reached by revolving the milled head in one and the same direction. It is immaterial in which direction the head is turned, just so this direction is not reversed just before a reading is taken. Since the zero point for the milled head alters with the direction in which it is rotated, the stirrup 23 carries two reference arrows, one for a clockwise, the other for a counterclockwise, direction of rotation of 21. The fractions of a per cent are read from the one or the other reference arrow according to the direction of rotation used. There is no endplay between disc and linear scale and no such correction is there necessary. The longitudinal movement $X$ of the indicator 22 is given by either of the following expressions:

$$
x=\frac{d \times l}{d_{2}-d_{1}} \quad \frac{s}{100}=\frac{r}{\operatorname{tang} \alpha}-\frac{s}{100}
$$

where $d$ is the diameter of the disk $18, l$ the length, $d_{2}$ the maximum, and $d_{1}$ the minimum, diameter of the cone, $s$ the slip in per cent, $r$ the radius of the disk 18 and $\alpha$ the angle which the generating line of the cone forms with the axis thereof. The scale 24 has equal divisions.

It is convenient to dimension the apparatus so that speeds of about 30 per cent below and about 15 per cent above synchronism can be measured with the same disk and cone, thus making the apparatus available for testing asynchronous generators and compensated motors. The same slipmeter will measure the slip of a motor with any number of poles provided the figure rotated by the motor under test has the proper number of elements. Various frequencies are taken care of by designing the synchronous motor to operate sufficiently well over the desired range of periodicities. The rotating figure can be observed directly through the slot in the stationary shield 19 or viewed by flashes of light projected through this slot.

It is, of course, desirable to keep the wear on the cone and disk down to a minimum. To this end, the disk should not be run longer than necessary and there should, of course, be no slip at all between the two. It has been found that good traction with a very small pressure is secured by using a ground brass cone and an aluminum disk. The pressure of the disk on the cone is regulated by suitably counterbalancing the carriage 26.

In the illustrations, the cone runs at the same speed as the four-pole motor which drives it. Since the disk has one slot, 1800 flashes will be produced at synchronism when operating on a 60 -cycle circuit. It is clear that in case of a 60-cycle four-pole motor, its slip can be ascertained with a figure having a single element, for instance, a single segmental spoke. This spoke will pass a fixed point of 1800 times a minute and will, therefore, appear stationary when viewed by 1800 flashes per minute. When the motor, and therefore the spoke, slips, the disk 18 must be caused to alter its speed correspondingly by turning the milled head 21 , thus again arresting the stroboscopic image. A sixpole, 60-cycle motor should carry three equally spaced segmental spokes, a triangle or the like. Tables II and III show the number of equally spaced elements required by figures revolved by 60 -cycle test motors of different number of poles in order that stationary stroboscopic images may be secured by a cone running at 1800 and $1200 \mathrm{rev}$. per min. respectively, and cooperating with a single-slot disk.

It will be found of advantage not to run the cone and disk too fast. A suitable speed can be readily selected by changing the gear ratio between motor and cone. A cone speed of 900 will be found satisfactory as to operation and wear; it requires the same set of shaft figures as an 1800 cone speed. The disk may have more than one slot. Any of the figures can be painted on a shaft end of the test motor or on a small disk firmly secured to the shaft. No great accuracy of outline or concentricity is required.

TABLE II

Shaft figures for use with 1800 flashes per minute

\begin{tabular}{|c|c|c|}
\hline $\begin{array}{l}\text { Poles of } \\
\text { test motor }\end{array}$ & $\begin{array}{l}\text { Synchronous } \\
\text { rev. per min. }\end{array}$ & $\begin{array}{c}\text { Number of } \\
\text { elements. }\end{array}$ \\
\hline 2 & 3600 & 1 \\
\hline 4 & 1800 & 1 \\
\hline 6 & 1200 & 3 \\
\hline 8 & 900 & 1 or 2 \\
\hline 10 & 720 & 5 \\
\hline 12 & 600 & 3 \\
\hline 14 & 514.3 & 7 \\
\hline 16 & 450 & 2 or 4 \\
\hline 18 & 400 & 9 and so on \\
\hline
\end{tabular}

TABLE III

Shaft figures for use with 1200 flashes per minute

\begin{tabular}{c|c|c}
\hline $\begin{array}{c}\text { Poles of } \\
\text { test motor }\end{array}$ & $\begin{array}{c}\text { Synchronous } \\
\text { rev. per min. }\end{array}$ & $\begin{array}{c}\text { Number of } \\
\text { elements }\end{array}$ \\
\hline 2 & 3600 & 1 \\
4 & 1800 & 2 \\
6 & 1200 & 1 \\
8 & 900 & 4 \\
10 & 720 & 5 \\
12 & 600 & 1 or 2 \\
14 & 514.3 & 7 \\
16 & 450 & 4 \\
18 & 400 & 3 and so on \\
\hline
\end{tabular}


Not only does this slipmeter permit of very accurate tests but it also saves time. In testing an induction motor with a speed counter, an attempt is generally made to keep the load constant for one minute. During this time, the voltage or the periodicity may vary and the load is sure to change unless such elaborate apparatus is employed as to make it impractical for everyday use. The volt, ampere and watt readings may, or may not, be taken at an average torque and the measured speed may, or may not, correspond to said readings. Furthermore, if the speed counter accuracy as referred to the rev. per min. is satisfactory, say 1 per cent, it nevertheless represents a large slip speed error corresponding to about 20 per cent for a 5 per cent slip, to 100 per cent for a 1 per cent slip, and so on. When using the slipmeter, readings of volts, amperes, watts, torque and speed are all taken simultaneously at the instant when the stroboscopic image becomes stationary for any particular setting of the brake; they are all exactly coordinate and the results incomparably more accurate. The slipmeter may be used to measure the slip of the smallest motors without vitiating the results because its use puts no load whatsoever on the machine.

When the test is made on a circuit, the periodicity of which is known to be practically constant, it is not necessary to use a frequency meter, but for particularly accurate work or where the line frequency is subject to material variation, frequency readings should be taken simultaneously with those already mentioned. A convenient form of wide range frequency meter consists of a synchronous motor adapted to operate satisfactorily over a wide range of periodicities coupled to a tachometer of the magneto type used in conjunction with a voltmeter scaled to read direct in cycles per second.

The Instantaneous Stroboscopic Speed Measuring Device permits the rate of revolution of any motor to be determined on the instant without putting any load on the machine whatsoever. It is intended for use in testing fractional horse power motors with any kind of a speed-torque characteristic. This apparatus consists of an adjustable-speed d-c. motor driving a magneto and a disk provided with one or more radial slots. The magneto cooperates with a voltmeter scaled to read direct in rev. per min. and the disk cooperates with a stationary shield provided with a single slot. This shield is so located that each disk slot coincides with the shield slot at one point of each revolution of the disk.

In making use of the apparatus, a geometrical figure is painted on one end of the shaft of the motor under test, or in case the shaft diameter is very small, a disk is firmly attached to the shaft, and observed through the stationary slot in the shield or illuminated by means of light flashes projected onto the shaft figure through the slot in question. The speed of the d-c. motor is then adjusted until the shaft figure appears stationary, when the voltmeter will show the test motor speed or a known multiple thereof.

It is best to select a shaft figure with a single element, and a single, segment-shaped spoke is generally used. The adjustable-speed motor is operated at 500 to 2000 rev. per min. being controlled in part by voltage and in part by field regulation, and drives the magneto direct. The voltmeter connected to the latter reads from 500 to $2000 \mathrm{rev}$. per min. The motor is geared 1:1 to the slotted disk and the stationary shield is supported by one of the bearings of the countershaft on which the revolving disk and its gear wheel are carried. The countershaft bearings are mounted on top of the d-c. motor. The revolving disk comprises two parts of the same outer diameter. One of these parts is provided with four equidistant radial slots and so keyed onto the countershaft as to make the disk slots coincide with open spaces between the spokes of the gear wheel keyed to the same shaft. The other part is provided with four equidistant slots to match the foregoing and with a fifth located midway between two of the four equidistant ones. This second part can be rotated about the shaft through 45 degrees. In one end position all four slots of both disks coincide and the result is a four-slot disk. In the other end position, the odd slot of one part coincides with one of the slots on the other part and the result is a oneslot disc. The two parts of the revolving disk are held in any desired relative position by means of screws.

Using the one-slot disk setting and a disk motor speed range of 500 to $2000 \mathrm{rev}$. per min., the number of flashes per minute can be varied from 500 to 2000 . Test motor speeds of 500 to 2000 rev. per min. can then be measured by adjusting the speed of the disk motor to produce the lowest speed, single stroboscopic image of the shaft figure. Under these conditions, the voltmeter will read direct in test motor rev. per min. By adjusting the disk motor speed to produce the lowest speed, double stroboscopic image, the voltmeter readings will record double the test motor speeds and test motor speeds of 250 to 1000 rev. per min. are thus covered.

Using the four-slot setting, the number of flashes for the same disk motor speed range can be carried from 2000 to 8000 per minute and test motor speeds of 2000 to 8000 rev. per min. measured by producing the lowest speed, single stroboscopic image of the shaft figure. In this case, the voltmeter reading must be multiplied by four in order to find the test motor speed.

If a range of 250 to 4000 is sufficient, then a oneand a two-slot disk is used, the change from one number to the other being made as in the case of the one- and the four-slot combination. For a two-slot disk, the voltmeter reading multiplier is two. It is of course easy to so arrange the two part disk that 1 , 2 and 4 slot disks can be produced by suitably displacing the two parts. 
The lower range limit can be reduced to 166.6 testmotor rev. per min. for 500 rev. per min. of the disk motor by adjusting for the lowest speed, triple stroboscopic image in which case the voltmeter reading must be multiplied by one-third to find the test-motor speed.

A tachometer of the magneto type which is regularly calibrated can be relied upon to give accurate enough results for most purposes over a range of 500 to 2000 magneto rev. per min., and readings may even be taken at less than 500 without fear of serious error. This little device therefore easily covers a test-motor speed range of 166 to 8000 rev. per min. and can be used to measure even lower speeds.

A possibility of error lies in the fact that single, double and triple stroboscopic images appear at several speeds, but these differ so greatly that a glance at the pulley of the test motor is in most cases sufficient to gage the order of magnitude of the speed being measured and to make sure that the stroboscopic image is secured at the lowest speed at which this image may be seen. The single spoke when viewed for instance by 500 flashes per minute is stationary and single at $500,1000,1500,2000$ rev. per min., stationary and double at 250, 750, $1250 \mathrm{rev}$. per min., and stationary and triple at $166.6,333.3,666.6 \mathrm{rev}$. per min., so that it is not very difficult to recognize the lowest speed of any series. Should confusion arise on this point it can be readily recognized and rectified when the speed curve is plotted.

\section{ILLUMINATION ITEMS \\ By the Lighting and Illumination Committee \\ THE FOOT-CANDLE METER ADAPTED TO AUTOMOBILE HEADLIGHT TESTS}

A number of states, particularly the more densely populated ones having complicated traffic problems, have automobile headlight legislation which specifies maximum and minimum candle power values at various angles, in order to eliminate objectionable glare and provide adequate road illumination. The ordinary photometric equipment available for making tests of this kind in the laboratory is usually very cumbersome and expensive. At the same time, many of the companies manufacturing and handling headlighting equipment have use for a portable and inexpensive photometric instrument enabling them to make fairly accurate candle power measurements under factory or service conditions. A number of devices is being marketed, suitable for this purpose. Of these the simplest and least expensive is the foot-candle meter. As commonly employed for industrial and similar commercial purposes it is graduated for illumination values somewhat higher than the minimum values ordinarily measured with automobile headlighting equipment. The regular foot-candle meter may, however, be simply and easily adapted to this special work by the addition of a $1 / 100$ point on the voltmeter scale as shown in Fig. 1, thereby permitting of readings as low as 100 candle power at 100 feet ahead of the car.

The explanation of the use of the foot-candle meter given in the book of instructions accompanying will doubtless eliminate all difficulty in its use. A number of foot-candle meters calibrated in this manner have been in use for some time with automobile and brass lamp manufacturers and are giving very satisfactory service.

In order to measure the candle power directed at a given angle from the headlights, it is merely necessary to hold the foot-candle meter with its face in a plane, normal to the beam at that point. With the voltmeter pointer set at the full scale arrow, the point at which the background and translucent spot appear equally bright will indicate the illumination received in footcandles as read from the scale. With a voltmeter pointer set at $1 / 10$ or $1 / 100$ scale, the values of illumination will be $1 / 10$ or $1 / 100$ respectively of the

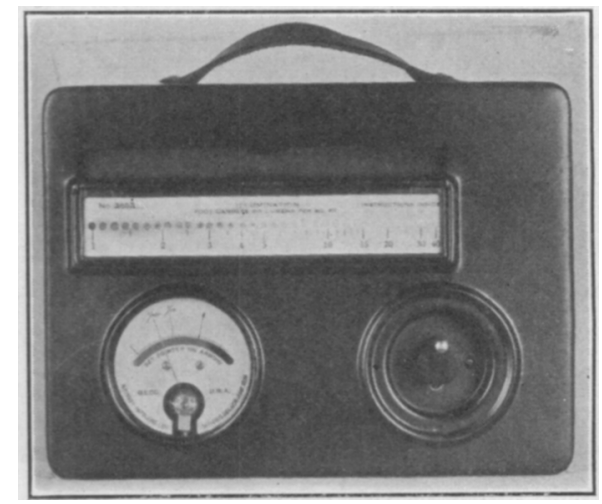

Fig. 1-With the Voltmeter Needle on the 1/100 Scale, at a Distance of 100 Ft. a Reading of 5 Foot-Candles Indicates an Illumination of 500 Candle Power in that Direction. This Scale is Most Useful in a Range from 200 to 2000 Candle Power

foot-candles indicated on the scale. The candle power in that direction will then be equal to the illumination value in foot-candles, multiplied by the square of the distance; for example, at 100 feet the multiplying factor would be 10,000 .

It will be noted that the scale of the meter runs from 1 to 40 foot-candles. Hence, at 100 feet, candle power values from 10,000 to 40,000 could be determined with the voltmeter pointer set at the full scale arrow. Inasmuch as it is easier to determine where the translucent spot and the background are equally bright when there are spots darker than the background below this point, it is desirable to use the full scale setting only for values down to, say, two foot-candles, corresponding to a candle power of 20,000 when measured at 100 feet. The $1 / 10$ scale similarly gives a range from 1000 to 40,000 but is most useful in the range from 2000 to 20,000 candle power as measured at 100 feet, that is the indications from 2 to 20 on the meter with the voltmeter pointer set at $1 / 10$ scale. Likewise, with the pointer set at $1 / 100$ scale, the 